\title{
Investigation and management of antenatally detected hydronephrosis
}

\author{
Karen Psooy, MD; ${ }^{*}$ John Pike, $M D^{\dagger}$
}

Can Urol Assoc J 2009;3(1):69-72

\section{Objective}

Antenatal hydronephrosis (ANH) affects approximately $1 \%$ of pregnancies. ${ }^{1}$ Controversies exist as to the postnatal investigation and management of these children. The CUA Guidelines Committee has reviewed the updated literature to provide recommendations.

\section{ANH as a predictor of postnatal pathology/need for further investigation}

ANH can represent a number of renal pathologies, the most common being uretero-pelvic junction obstruction (UPJO), vesico-ureteral reflux (VUR), posterior urethral valves (PUV) and ureteralvesical junction obstruction (UVJO). ${ }^{2}$ AnteriorPosterior Diameter (APD) is the most common method for reporting antenatal $\mathrm{ANH}$. The grades of $\mathrm{ANH}$ have been defined as follows (Table 1). ${ }^{2}$

\section{Postnatal interventions, investigations and timing}

\section{Prophylactic antibiotics}

The role of prophylactic antibiotics in children with $\mathrm{ANH}$ who are awaiting completion of post- natal investigations is controversial. Children with ANH have been shown to have a significantly higher risk of developing a urinary tract infection (UTI) than controls. ${ }^{3}$ While it is believed that prophylactic antibiotics will prevent urinary tract infections in children with VUR, it has yet to be proven. ${ }^{4}$ Therefore, some recommend institution of prophylactic antibiotics at birth:, ${ }^{1,5}$ others instead recommend a low threshold for investigation and treatment of a suspected UTI. ${ }^{3}$ Commonly used prophylaxes in the neonate include amoxicillin' $50 \mathrm{mg}$ per day and cephalexin ${ }^{6} 50 \mathrm{mg}$ per day. Trimethoprim-sulfamatholxazole ${ }^{1}$ and nitrofurantoin $^{7}$ should NOT be used in the neonate.

Renal ultrasound (RUS)

RUS allows reassessment of the grade of $\mathrm{ANH}$ and may point toward a distinct etiology.

\section{Timing}

All children with $\mathrm{ANH}$ should have a postnatal RUS. Children with bilateral severe $\mathrm{ANH}$, and children with ANH of any degree in a solitary kidney should have a postnatal RUS prior to discharge from hospital. ${ }^{2}$ However, ideally, the first postnatal RUS should not be performed within the first 48-72 hours, as relatively low urine output during this period may underestimate the degree of

\begin{tabular}{|c|c|c|c|}
\hline ANH grade & $\begin{array}{l}\text { APD second } \\
\text { trimester, mm }\end{array}$ & $\begin{array}{l}\text { APD third trimester, } \\
\qquad \mathrm{mm}\end{array}$ & $\begin{array}{c}\text { \% with postnatal } \\
\text { pathology }\end{array}$ \\
\hline Mild & $\leq 7$ & $\leq 9$ & 12 \\
\hline Moderate & $7-10$ & $9-15$ & 45 \\
\hline Severe & $>10$ & $>15$ & 88 \\
\hline All & - & - & 36 \\
\hline
\end{tabular}


hydronephrosis. ${ }^{1}$ Therefore, in this situation, if the RUS is normal and the serum creatinine is dropping appropriately, a repeat RUS should be performed the following week. The remainder of children with ANH should have a RUS performed between 7 and 30 days. ${ }^{1,2}$

\section{Grading}

Postnatally, a commonly used system to grade hydronephrosis is that of the Society of Fetal Urology (SFU). The SFU grading system is as follows. ${ }^{8}$

\section{Grade Characteristics of central renal echo complex}

$0 \quad$ Closely apposed

1 Slight separation

2 Further separation; one or few calyces may be visualized

3 Pelviectasis and fluid filled calyces seen throughout kidney

$4 \quad$ Grade 3 with parenchyma over calyces thinned

Only grades 3 and 4 are felt to be clinically significant with respect to obstruction. ${ }^{1}$

All grades of $\mathrm{ANH}$ have an increased risk of associated VUR (8.5-15\%). ${ }^{2,9}$ Unlike with obstruction, the grade of $\mathrm{ANH}$ does not positively correlate with the likeliness of diagnosing VUR. ${ }^{2}$

\section{Voiding cystourethrogram (VCUG)}

VCUG is the preferred investigation to diagnose and grade VUR. It also allows for anatomical assessment of the urethra (to rule out PUV) and bladder.

\section{Timing and selection}

An urgent VCUG before discharge from hospital is essential in any child with ANH where there is a prenatal concern of bladder outlet obstruction (ex: PUV). ${ }^{1}$ Other absolute indications for a VCUG to investigate $\mathrm{ANH}$ include: severe bilateral hydronephrosis (urgent), suspected infravesical obstruction (urgent), dilated ureter, duplex kidney, abnormal echogenicity and abnormal bladder (less urgently, around one month of age). ${ }^{1}$

Controversy exists around whether children with SFU 0-2 on postnatal RUS should get a VCUG. Reflux will be found in approximately $15 \%{ }^{9}$ of these children in comparison to $1 \%$ in the general population. ${ }^{2}$ What is debated is whether this VUR is clinically significant and in need of being diagnosed.

Children with persistent moderate or severe hydronephrosis should be investigated with a VCUG. ${ }^{2}$ If VUR is found, it will be of high grade. Additionally, if the refluxed contrast drains freely, concomitant UPJO can be ruled out.

\section{Diuretic renography (DTPA or MAG-3)}

DTPA or MAG-3 may help to diagnose, or rule out obstructive uropathy. Unfortunately, diuretic renography is not completely reliable in infants. ${ }^{1,10}$ However, if the scan suggests obstruction, differential function is a useful tool to determine the need for surgical intervention. ${ }^{1}$

\section{Timing and selection}

SFU grade 3-4 hydronephrosis that is not explained by VUR should be investigated further with diuretic renography at 4-6 weeks of age. ${ }^{1}$

\section{Need for further investigations following completion of initial investigations}

If the initial postnatal RUS shows only isolated SFU 0-2 ANH, and a VCUG is performed, showing no VUR, it is unlikely that the hydronephrosis will become significant. However, some advocate another ultrasound at 6 months. ${ }^{5}$ While many physicians (and likely parents) would like to see improvement, or confirm stability with a repeat RUS at 6 months, particularly in those with SFU $1-2$, it is not clear if this is necessary. However, the event of a systemic urinary tract infection should stimulate re-evaluation, which may include diuretic renography. ${ }^{5}$

If the initial postnatal RUS shows SFU 0-2 ANH and one elects not to perform a VCUG, reflux may still exist and there is a need to ensure that the parents are vigilant regarding prompt assessment if signs of a UTI develop. ${ }^{6}$ The event of a systemic UTI should stimulate a VCUG being performed. The role for further RUSs in these children is also unclear.

SFU III-IV ANH in the absence of VUR requires ongoing observation with serial RUSs and judicious use of diuretic renography. The details of 
such observation are beyond the scope of this guideline. These children should be referred to a pediatric urologist.

The follow-up and management of diagnosed UPJO and VUR are beyond the scope of this guideline.

\section{Summary}

1. All ANH should be investigated with a postnatal RUS. ${ }^{2}$

- Level 1 Evidence: Grade A Recommendation

2. The role of prophylactic antibiotics initiated at birth is controversial.

- Grade D Recommendation
3. The need to further investigate mild postnatal hydronephrosis (SFU 0-II) with a VCUG is controversial, and depends on the physician's attitude toward diagnosing asymptomatic VUR.

- Grade D Recommendation

4. The need to reassess mild postnatal hydronephrosis with a second RUS is unclear, but does provide reassurance to the physician and parent if the $\mathrm{ANH}$ remains stable or improves.

- Level 4 Evidence: Grade C Recommendation

5. Persistent moderate or severe hydronephrosis (SFU III-IV) should be investigated with a VCUG, followed by diuretic renography if the hydronephrosis cannot be explained by VUR.'

- Level 4 Evidence: Grade C Recommendation

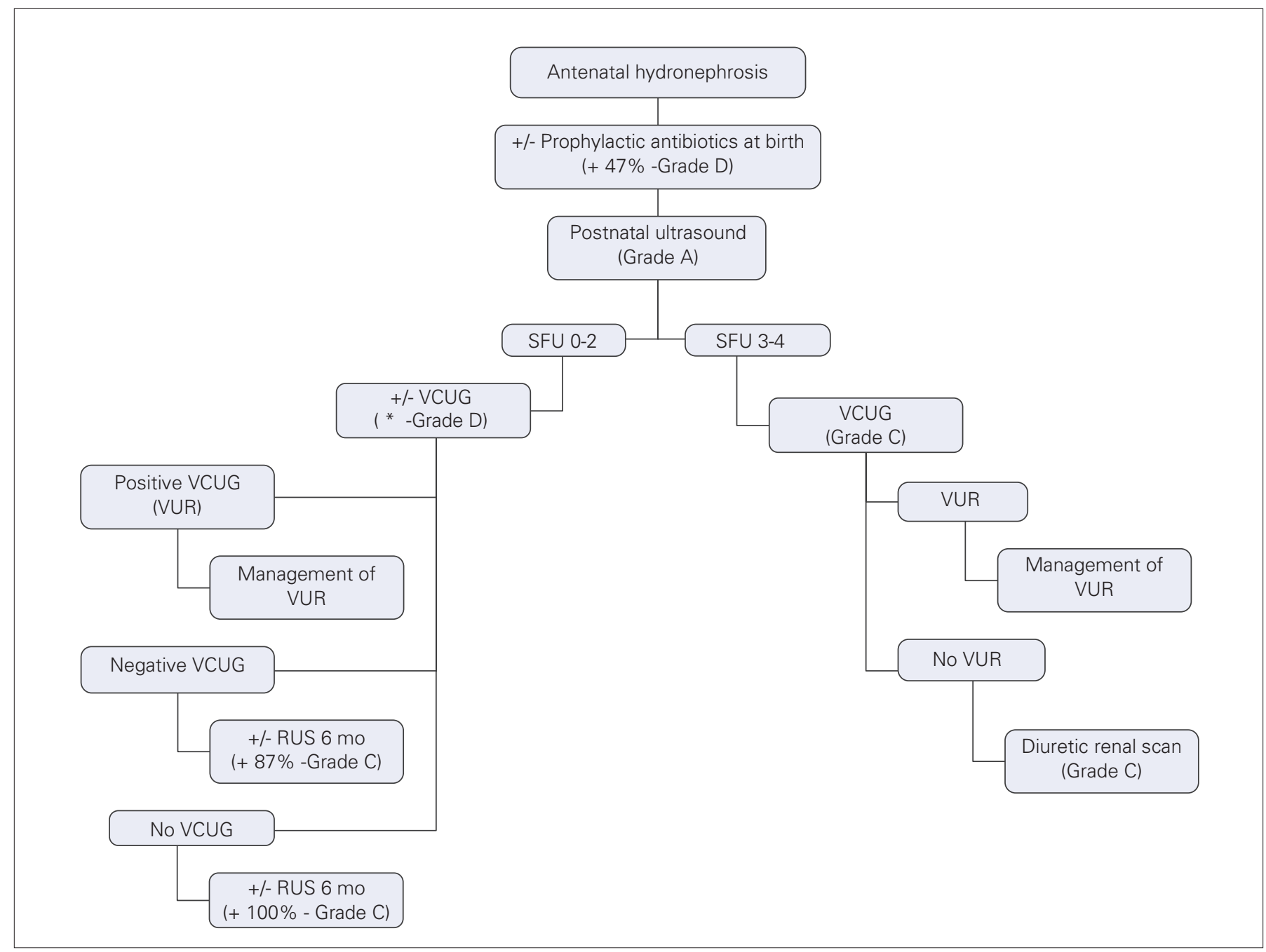

Fig 1. Summary flow chart of Levels of Evidence and Grade recommendations for evaluating antenatal hydronephrosis. *93\% would not order a voiding cystourethrogram (VCUG) if Society of Fetal Urology (SFU) 0; 80\% would not order a VCUG for SFU I; 73\% would order or consider ordering a VCUG for SFU II. VUR = vesico-ureteral reflux; RUS = renal ultrasound. 
A summary flow chart is provided (Fig. 1). To assist in the decision-making of controversial areas, members of the Pediatric Urologists of Canada were surveyed in 2007 to determine most common practice patterns. The results, based on the responses of 15 Canadian members, are included in the flow chart.

From the *Division of Pediatric Urology, Winnipeg Children's Hospital, Winnipeg, Man., and the †Children's Hospital of Eastern Ontario, Ottawa, Ont.

This article has been peer reviewed.

Competing interests: None declared.

\section{References}

1. Belarmino JM, Kogan BA. Management of neonatal hydronephrosis. Early Hum Dev 2006:82:9-14

2. Lee $R S$, Cendron $M$, Kinnamon $D D$, et al. Antenatal hydronephrosis as a predictor of postnatal outcome: a meta-analysis. Pediatr 2006;118:586-93.
3. Harding $\downarrow$, Malone PSJ, Wellesley DG. Antenatal minimal hydronephrosis: is its followup an unnecessary cause of concern? Prenat Diagn 1999;19:701-5.

4. Garin EH, Olavarria F, Nieto VG, et al. Clinical significance of primary vesicoureteric reflux and urinary antibiotic prophylaxis after acute pyelonephritis: a multicenter, randomized, controlled study. Pediatr 2006;1 17:626-32.

5. Aksu $N$, Yavascan 0 , Kangin $M$, et al. Postnatal management of infants with antenatally detected hydronephrosis. Pediatr Nephrol 2005;20:1253-9.

6. Hallerstein S, Nickell E. Prophylactic antibiotics in children at risk for urinary tract infection. Pediatr Nephrol 2002;17:506-10.

7. Consult MD. Available: www.mdconsult.com (accessed 2008 Mar 18).

8. Fernbach SK, Maizels M, Conway JJ. Ultrasound grading of hydronephrosis: introduction to the system used by the Society of Fetal Urology. Pediatr Radiol 1993;23:478-80.

9. Gloor JM, Ramsey PS, Ogburn PL Jr, et al. J Matern Fetal Neonatal Med 2002;12:196200.

10. Koff SA, Binkovitz L, Coley B, et al. Renal pelvis volume during diuresis in children with hydronephrosis: implications for diagnosing obstruction with diuretic renography. J Urol 2005;174:303-7.

Correspondence: Dr. Karen Psooy, University of Manitoba, GE446 - 820 Sherbrook St., Winnipeg MB R3A 1R9; kpsooy@hsc.mb.ca 